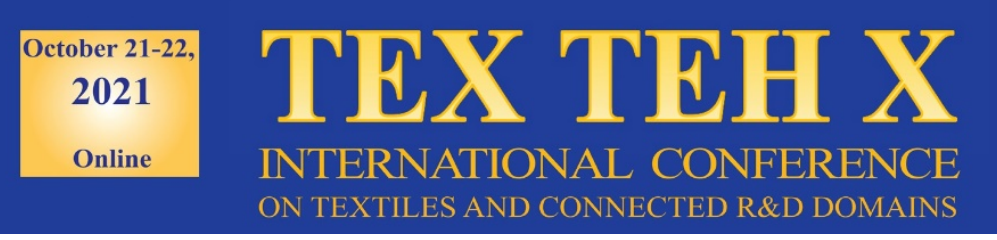

\title{
INTEGRATION OF MAGNETIC MATERIALS WITH ACTUATOR ROLE ON TEXTILE SUPPORTS
}

\author{
DOI: $10.35530 /$ TT.2021.45
}

\author{
C. Grosu*, R.M. Aileni, S. Olaru
}

National Research Development Institute for Textiles and Leather, Romania

(E-mail: cristina.grosu@incdtp.ro; raluca.aileni@incdtp.ro; sabina.olaru@incdtp.ro)

\begin{abstract}
Magnetic textile materials represent a new category of smart materials, whose properties are obtained either by adding magnetic materials during the technological processes of obtaining fibres and yarns, either by applying some magnetic materials on textile surfaces during the chemical finishing processes (electroless plating, electroplating, magnetron sputtering). Therefore, by adding magnetic nano powders in the spinning solution, fibres with magnetic properties are obtained, by adding metallic fibres, with magnetic properties, during the spinning process, magnetic yarns are obtained, and by the insertion of a certain percent of metallic/magnetic yarns during the weaving or knitting process, textile materials with magnetic properties are obtained. Thus, magnetic textile materials will possess the uniqueness of a textile structure due to specific features as flexibility, breathability or lightweight, but at the same time, also the magnetic properties necessary in multiple applications such as magnetic sensors, actuators and electromagnetic shielding used in technical applications for defence, automotive and aerospace.
\end{abstract}

Keywords: actuators, composites, filler, functionalization, magnetic materials, textiles

\section{INTRODUCTION}

Magnetic textiles belong to the group of smart textiles, as independently of their natural textile features, they are characterized by new magnetic properties which depend on the type of magnetic material (the filler) included in the textile matrix, as well as on the filling degree [1]. For the magnetic functionalization of the textile materials, are usually used composites based on magnetic materials such as iron oxide nanoparticles $\left(\mathrm{Fe}_{2} \mathrm{O}_{3}\right.$ or $\left.\mathrm{Fe}_{3} \mathrm{O}_{4}\right)$ combined with conductive polymers (polypyrrole (PPY), polyaniline (PANI), polythiophene (PTH)). Inherently, conductive polymers such as PPY or PANI, known as "synthetic metals", are unique due to their electrical properties similar to those of metals, while maintaining the mechanical characteristics of polymers [2-4]. The resulted composite materials, consisting of two or more components with magnetic and electrical properties, gain significantly different electromagnetic, physical and chemical properties, as compared with the properties of initial materials. Thus, the added magnetic component allows the responses of the composite material under the influence of an external magnetic field $[5,6]$ and is very useful in medical applications for drug delivery.

The parameters such as dielectric permittivity, magnetic permeability and electrical conductivity define the electromagnetic properties of a textile material [7].

This research paper presents a study of the magnetic textile materials - focused on the obtaining processes, their magnetic properties and applications. 


\section{OBTAINING PROCESSES OF MAGNETIC TEXTILE MATERIALS}

Conventional textiles are generally obtained from non-magnetic and non-conductive polymeric materials, which means that they are magnetically negative. Table 1 shows the values of magnetic susceptibility $(\chi)$, for some reference raw textile materials.

Table 1. Magnetic susceptibility of some textile fibres [7]

\begin{tabular}{|c|c|}
\hline Material & Magnetic susceptibility $(\chi)$ \\
\hline Ethylene & $-10.3 \times 10^{-6}$ \\
\hline Polypropylene & $-10.1 \times 10^{-6}$ \\
\hline Fluorine & $-47.8 \times 10^{-6}$ \\
\hline Polyester & $-6.53 \times 10^{-6}$ \\
\hline Nylon 66 & $-9.55 \times 10^{-6}$ \\
\hline
\end{tabular}

Magnetic functionalization of the textile materials can be achieved by:

- the introduction of some materials with special properties during the obtaining process of fibre/yarn/material;

- the deposition of some materials with specific characteristics on the fibre/yarn/material surface during the finishing process $[7,8]$.

Therefore, magnetic functionalization of fibres, yarns and 2D and 3D textile surfaces can be obtained:

a) during the fabrication process of the fibres;

b) during the spinning process of the yarns;

c) during the obtaining process of braided, woven, non-woven and knitted materials;

d) during the finishing process of fibres, yarns and textile structures $[7,9,10]$.

\subsection{Fibres functionalization}

During the spinning process of the textile fibres, metallic fibres, carbon/ graphite fibres, or intrinsic conductive polymeric materials, with intrinsic electromagnetic functionalities, can be used to replace partially or totally the conventional fibrous textile material. The most exploited raw material for obtaining magnetic textile fibres is cellulose, in the solution of which is possible to add powders with magnetic properties, such as iron, cobalt, nickel and different ferrites, by mixing during the spinning process [7,11].

Chemical finishing, in the case of conventional fibres that lack electromagnetic properties, allows the deposition on their surface of some metallic coatings or powders by different procedures, such as electroless plating, electroplating, magnetron sputtering, in situ polymerization, etc. [7]. The most exploited textile fibres suitable for magnetic functionalization by chemical finishing are natural vegetal fibres, like cotton, with high cellulose content, known for its important role in fixing and stabilizing the binder, with a high degree of hygroscopicity and retention of aqueous substances, but also new as mango fibres, etc. [12-15].

\subsection{Yarns functionalization}

The introduction of fibres with electromagnetic properties during the spinning process of yarns leads to magnetic yarns. Obtaining these yarns by processes like blending or enveloping, necessitates particular attention, especially regarding the ratio between the magnetic/metallic fibres and the conventional ones, knowing that metallic fibres are not suitable to be used alone for weaving because of their low elongation and poor toughness [7]. 
By inserting a microfilament with electromagnetic properties in the yarn structure, there could be obtained the following yarns with magnetic properties: i) yarns obtained by enveloping the exterior of a metallic microfilament with a fibrous layer; ii) twisted yarn obtained by twisting together a metallic microfilament with a conventional textile yarn; iii) plied yarn obtained by parallel feeding of the conventional textile yarn and the metallic filament [16].

Chemical finishing is a deposition process onto the yarn surface of a metallic coating or powder by processes like electroless plating, electroplating, magnetron sputtering, etc., to obtain the targeted magnetic properties $[7,17]$.

\subsection{Textile surfaces functionalization}

During the weaving or knitting process of textile structures, the metallic yarn cannot be used as unicomponent element, due to its poor elongation and flexibility, necessitating additional processing steps like blending or enveloping with conventional fibres. Thus, there can be obtained functionalized yarns with magnetic properties, adequate for direct feeding of weaving or knitting machines, in different ratios with conventional yarns [7]. Several experimental reports have shown that both in the case of rectilinear or circular knitting machines, as well as in the case of classical weaving machines, feeding of yarns with metallic microfilament content was possible, in the same time establishing the relationship between the feeding ratio of metallic yarns and the electromagnetic shielding effectiveness [16].

Chemical finishing techniques, like solution impregnation, coating or scraping, represent the last step in the fabrication process of textile material and implies the addition of chemical products to the textile materials, their aspect remaining generally unchanged after these procedures [8].

Impregnation with a solution containing ferromagnetic microfilaments proved to be unsuitable in the case of functionalizing textile materials intended for shielding electromagnetic waves. By impregnation of a $100 \%$ cotton fabric with a $20 \%$ polyurethane solution, containing ferromagnetic filaments, an uneven distribution onto the fabric surface of the ferromagnetic microfilaments was obtained. Fibre agglomerations appeared even since the blending step with the polyurethane solution. Solving the problem of uneven distribution through solution impregnation in two steps proved to be inefficient in terms of the filling degree of the composite material, which increased proportionally with the polyurethane solution concentration [16].

In the case of metallic nanoparticles easily oxidized by air or aqueous solutions (e.g., nano copper), it is recommended the addition of a small quantity of ascorbic acid (Vitamin C) in the polymeric matrix of the dopped solution with ferromagnetic filaments/powders [18].

The coating with neo dim magnetic microparticles onto textile substrates obtained by different technologies (knitting or weaving), revealed higher performances for the composite based on knitted structure compared to the woven structure, due to the higher energy present on the surface, due to the higher wetting capacity and rubbing coefficient, leading so to higher retention of magnetic powder [10].

Significant magnetic performances have been obtained by coating cotton with barium ferrite (BaFe12O19) doped aniline solution, resulting in a new composite material, with electromagnetic properties with particular values of remanent magnetization of $0.54 \mathrm{emu} / \mathrm{g}$, as well as coercive value of $4700 \mathrm{G}$, under ambient conditions [19].

Scraper coating of some woven textile materials with solutions dopped with hard magnetic powders of barium hexaferrite $\left(\mathrm{BaFe}_{12} \mathrm{O}_{19}\right)$, demonstrated that the magnetic performances increase directly proportional to the percent of magnetic powder from the coating solution. For a more even deposition onto the textile surface, it is recommended that 
the size of selected ferromagnetic powders to be at nano level [20]. Similar conclusions were elaborated, that the increase of the mass, thickness and air permeability of fabric will generate the decreasing of the conductivity, a fact explained by the decreased concentration of the magnetic nanoparticles onto the fabric surface, when multivariate regression analysis was applied [21].

Magnetron sputtering allows the deposition metallic layers, with nanometre thicknesses onto the textile structure surfaces. The tests have shown that by copper deposition onto the textile structure surfaces, the electromagnetic shielding effectiveness of textile materials grew significantly [22].

Plasma treatment at atmospheric pressure proved to be superior to conventional cleaning, by improving the hydrophily. The process is more friendly with the environment compared to conventional procedures, which implies the use of higher quantities of water and various chemical substances $[23,24]$.

\section{APPLICATIONS OF MAGNETIC TEXTILE MATERIALS}

Due to the fundamental properties exhibited by the magnetic textiles, such as electrical conductivity, dielectric permittivity and magnetic permeability these are exploited in a large number of applications in the field of aerospace, automotive, medicine and smart clothing (sport, protection) [18].

Composites obtained by introducing ferromagnetic nano powders into the fibres spinning solution are also known as electromagnetic composites, due to their multiple applications in structures of microelectronic and nano-electronic micro-mechanical systems (MEMS) and bio-micro-mechanical systems (MioMEMS) [1].

Magnetic textiles exhibit a great potential for applications in the fields of medical devices (actuators and magnetic sensors). Magneto-active fabrics (MAFs), as magnetic dielectric tissues, obtained through soaking with silicone oil and iron oxide microfibers, represent a promising candidate for the fabrication of health monitoring systems and electromagnetic radiation absorbers [25].

Luminescent magnetic fibres obtained from the modification of the lyocell fibres through the NMNO ( $N$-methyl morpholine-N-oxide) method are successfully used for the production of threads and yarns. Under UV light irradiation, this novel fibre glows and exhibits magnetization, being an innovative method of intellectual rights protection in the fashion industry, because the original garments become almost impossible to counterfeit [26].

\section{CONCLUSIONS}

Factors that influence the magnetic functionalization process of conventional textile materials, by integration of magnetic materials on the textile structure surfaces, are as follows:

- Nature of the textile raw material: cotton fibre is the most frequently used for the substrates of the composite materials designed with magnetic properties based on classical finishing processes with dispersions or pastes-based water. This is due to the multiple polar cellulose groups present on the cotton fibre, which gives its high hydrophilicity and an excellent wetting capacity, but also because improves the comfort properties of the resulted composite material. Even if synthetic fibres, such as polyamide and polyester, although possess polar groups, cannot absorb large quantities of water, due to their advanced crystallinity $[2,10,16,18,19,22]$, but can be used successfully in case of using the coating technologies such as electroless plating, electroplating or magnetron sputtering. 
- Obtaining process of the textile substrate: there are only a few studies regarding the obtaining of composite materials with magnetic properties, using knitted structures as substrates, compared with the using of woven structures. Knitwears, especially the 3D ones, possess a high potential for exploitation in this direction, due to high breathability given by the controllable porous structure, due to the controllable cross-section geometry by binding the external layers with yarns or other knitted structures having electromagnetic properties.

The high potential of the knitwears was also confirmed by several studies that compared the magnetic properties obtained by depositing under similar conditions of the solutions containing ferromagnetic materials on knitted and woven substrates, respectively $[2,7,10]$.

Mainly, for magnetic composites development are used conductive polymers such as PPY, PANI, PTH mixed with magnetic nanoparticles. These composites obtained have numerous applications for actuators [2-4,27].

The higher the concentration of magnetic powder dopped in the coating solution of the textile substrate, the higher the magnetic induction intensity of the composite material $[10,19,20]$.

To improve the electromagnetic properties of a textile structure it is possible to simultaneously use: the insertion of conductive yarns during the weaving/knitting process, followed by the deposition onto the surface material of a thin layer containing ferromagnetic nanoparticles $[22,28]$.

\section{ACKNOWLEDGEMENTS}

This work was supported through the National Nucleu Programme, with the support of MEC, Contract PN 191701 01, project title: "Composite materials with electroconductive properties, based on 3D polymeric array for sensorial monitoring systems and electromagnetic waves attenuation (3D ELECTROTEX)”.

\section{REFERENCES}

[1] Rubacha, M., Zieba, J., Magnetic Textile Elements, In: Fibres \& Textiles in Eastern Europe, 2006, 14, 59, 49-53

[2] Loghin, C., Cianga, L., Ciobanu, L., Cianga, I., Yagci, Y., Nicolaiov, P., Textile composites based on conductive polymers, In: Buletinul Institutului Politehnic din Iasi, 2010, 57-66

[3] Teslaru, T.L., Caracterizarea filmelor de politiofen obținute în reactori cu plasmă la presiune atmosferică, Iasi, 2015

[4] Kongahage, D., Foroughi, J., Actuator materials: review on recent advances and future outlook for smart textiles, In: Fibers, 2019, 1-24

[5] Loghin, C., Composite textile structures for protective systems against electromagnetic radiations, Iasi, 2007

[6] INCD pentru Tehnologii Izotropice si Moleculare, Cresterea capacitatii de transfer tehnologic si de cunoastere a INCDTIM in domeniul Bioeconomiei, Cluj Napoca, 2018

[7] Xiao, H., Shi, M., Chen, J., Electromagnetic Function Textiles, In: Electromagnetic Materials, IntechOpen, 2019, 1-32

[8] Kumar, R.S., Sundaresan, S., Advances in the dyeing and finishing of technical textiles, In: M.L. Gulrajani, Advances in the dyeing and finishing of technical textiles, Woodhead Publishing, 2013, 270

[9] Ehrmann, A., Blachowicz, T., Magnetic Yarns, Fabrics, and Coatings, In: Examination of Textiles with Mathematical and Physical Methods, Springer, 2016, 31-46, https://doi.org/10.1007/978-3-31947408-3_3

[10] Zhou, Y., Zhu, W., Zhang, L., Gong, J., Zhao, D., Liu, M.,Sun, Y., Magnetic properties of smart textile fabrics through a coating method with $N d F e B$ flake-like microparticles, In: Journal of 
Engineered

fibers

and

fabrics,

2019,

14 ,

https://journals.sagepub.com/doi/10.1177/1558925019865708

[11] Rubacha, M., Zieba, J., Magnetic Cellulose Fibres and Their Application in Textronics, In: Fibres \& Textiles in Eastern Europe, 2007, 15, 5-6, 101-104

[12] Small, A.C., Johnston, J., Novel hybrid materials of magnetic nanoparticles and cellulose fibers, In: Journal of Colloid and Interface Science, 2008, https://doi.org/10.1016/j.jcis.2008.11.038

[13] Souza Junior, F.G., da Silva, A.M., de Oliveira, G.E., Costa, R.M., Fernandes, E.R., Pereira, E.D., Conducting and magnetic mango fibers, In: Industrial Crops and Products, 68 Special Issue, 2014, 97104, https://doi.org/10.1016/j.indcrop.2014.09.032

[14] Frydrysiak, M., Grosu, M. C., Zieba, J., Lupu, I.G., Textile magnetic yarns - research and simulation, In: Industria Textila, 2015, 66, 6, 322-328

[15] Blachowicz, T., Ehrmann, A., Simulation on magnetic coatings on textile fibers, In: Journal of Physics, 2016, http://doi.org/10.1088/1742-6596/738/1/012057

[16] Universitatea Tehnică "Gheorghe Asachi", Sisteme mecatronice inteligente pentru echipamente textile, Iasi, 2010

[17] Grosu, M.C., Lupu, I.G., Cramariuc, O., Hogas, H.I., Fabrication and characterization of magnetic cotton yarns for textile applications, In: The Journal of the Textile Institute, 2018, 109, 10, 1348-1359, https://doi.org/10.1080/00405000.2018.1423935

[18] Hassabo, A.G., El-Naggar, M.E., Mohamed, A.L., Hebeish, A.A., Development of multifunctional modified cotton fabric with tri-component nanoparticles of silver, copper and zinc oxide, In: Carbohydrate Polymers, 2019, 144-156

[19] Onar, N., Akșit, A.C., Ebeoglugil, M.F., Conductivity and magnetic properties of coated fabrics with barrium ferrite doped aniline solution, In: International Technical Textiles Congress, Istambul, ResearchGate, 2007, 200-208

[20] Grosu, M.C., Lupu, I.G., Avram, D., Tudorache, F., Magnetic woven fabrics - physical and magnetic properties, In: Annals of the University of Oradea. Fascicle of Textiles, Leatherwork, 2015, 43-48

[21] Aileni, R.M., Albici, S., Chiriac, L., Sandulache, I.M., Multivariate regression analysis of the 3D composites with electroconductive, In: Industria Textila, 2020, 71, 4, 334-339, https://doi.org/10.35530/IT.071.04.1767

[22] Rădulescu, I.R., Surdu, L., Mitu, B., Morari, C., Costea, M., Golovanov, N., Conductive textile structures and their contribution to electromagnetic shielding effectiveness, In Industria Textila, 2020, 71, 5, 432-437, https://doi.org/10.35530/IT.071.05.1783

[23] Kan, C.-w., Lam, C.-f., Atmospheric pressure plasma treatment for grey cotton knitted fabric, In: Polymers, 2018, 1-16

[24] Aileni, R.M., Albici, S., Dinca, L., Surdu, L., Bivariate analysis of the hydrophobic textiles obtained by plasma treatment, In: Industria Textila, 2019, 70, 6, 527-532, https://doi.org/ 10.35530/IT.070.06.1476

[25] Bunoiu, M., Anitas, E. M., Pascu, G., Chirigiu, L. M., Bica, I., Electrical and Mangetodielectric Properties of Magneto-Active Fabrics for Electromagnetic Shielding and Health Monitoring, In: International Journal of Molecular Sciences, 2021, 21, 13, https://doi.org/10.3390/ijms21134785

[26] Skwierczynska, M., Runowski, M., Goderski, S., Szczytko, J., Rybusinski, J., Kulpinski, P., Lis, S., Luminiescent-Magnetic Cellulose Fibers, Modified with lanthanide- Doped Core/ Shell Nanostructures, In: ACS OMEGA, 2018, 3, 10393-10390, https://doi.org/10.1021/acsomega.8b00965 [27] Pandey, D.N., Basu, A., Kumar, P., Electro Conductive Knitted Structure: An Over View, In: Emerging Trends in Engineering \& Technology, 2016, 261-265

[28] Maziz, A., Concas, A., Khaldi, A., Stalhald, J., Persson, N.-K., Jager, E., Knitting and weaving artificial muscles, In: Applied Science and Engineering, 2017, 1-11 